J. Lundén and V. Koivunen, "Scaled conjugate gradient method for radar pulse modulation estimation," in Proceedings of the IEEE International Conference on Acoustics, Speech, and Signal Processing (ICASSP), Honolulu, HI, USA, April 15-20, 2007, vol. 2, pp. 297-300.

(C) 2007 IEEE. Reprinted with permission.

This material is posted here with permission of the IEEE. Such permission of the IEEE does not in any way imply IEEE endorsement of any of the Helsinki University of Technology's products or services. Internal or personal use of this material is permitted. However, permission to reprint/republish this material for advertising or promotional purposes or for creating new collective works for resale or redistribution must be obtained from the IEEE by writing to pubs-permissions@ieee.org.

By choosing to view this material, you agree to all provisions of the copyright laws protecting it. 


\title{
SCALED CONJUGATE GRADIENT METHOD FOR RADAR PULSE MODULATION ESTIMATION
}

\author{
Jarmo Lundén and Visa Koivunen \\ Signal Processing Laboratory, SMARAD CoE \\ Helsinki University of Technology \\ P.O. Box 3000, FI-02015 TKK, Finland
}

\begin{abstract}
This paper addresses the problem of estimating a common modulation from a group of intercepted radar pulses. Estimated modulation profile operates as the basis for specific emitter identification (SEI). A robust M-estimation technique using scaled conjugate gradient algorithm for improving the frequency alignment of the pulses is proposed. In addition, postprocessing of the estimated modulation profiles for identification is considered. Simulation experiments are conducted in order to compare the performance with previously proposed methods. Results show that the proposed robust M-estimation technique provides improved performance at low signal-to-noise ratio regime due to better frequency alignment of the intercepted pulses.
\end{abstract}

Index Terms - M-estimation, radar, robustness, scaled conjugate gradient, specific emitter identification

\section{INTRODUCTION}

Specific emitter identification is an important task in spectrum monitoring and electronic warfare. It is a crucial component in threat detection and analysis in electronic support (ES). Both intentional and unintentional modulations in the radar signals provide important information to perform SEI. Different intrapulse information based approaches have been proposed for SEI during the past few years. Intrapulse information based clustering and determination of the number of emitters from a set of collected pulses using an information theoretic criterion has been proposed in [1]. Feature based approach for SEI has been proposed in [2]. Time-frequency distribution based approach was considered in [3].

In [4] a maximum likelihood (ML) estimation method was proposed for estimating a common modulation from a group of intercepted pulses. Furthermore, hypothesis testing based technique was proposed for identifying radar emitters. In order to robustify the process, an M-estimation technique for estimating the common modulation from a group of intercepted radar pulses was proposed in [5]. The technique was shown to provide better tolerance against preprocessing errors than the ML estimator. In this work, the Mestimator using equal weights within pulses [5] is extended by using scaled conjugate gradient (SCG) algorithm to improve the frequency alignment of the pulses. In addition, a postprocessing method is introduced in order to further improve the frequency alignment for identification purposes. Simulation results indicate that improved frequency alignment and thus better estimation performance is achieved compared to the previous ML and M-estimation methods.

This work has been funded by the Finnish Defence Forces Technical Research Centre.
The paper is organized as follows. Section 2 presents the signal model. Section 3 explains the preprocessing stage and Section 4 introduces the proposed M-estimation algorithm. Section 5 describes the postprocessing stage. The simulation results are presented in Section 6. Finally, Section 7 gives the conclusion.

\section{SIGNAL MODEL}

In the following it is assumed that the sampling rate is high enough to record the pulse modulations faithfully. Moreover, it is assumed that each intercepted pulse consists of $N_{S}$ complex samples from a single mode of a radar including a buffer of samples recorded before and after the pulse.

The complex pulse vectors $\boldsymbol{y}_{k}$ from the same emitter are assumed to be given by [4]

$$
\boldsymbol{y}_{k}=A_{k} \boldsymbol{\mathcal { T }}\left(\tau_{k}\right) \boldsymbol{\Omega}\left(\omega_{k}\right) \boldsymbol{\mu}+\boldsymbol{\epsilon}_{k}, \quad k=1, \ldots, N_{P},
$$

where $A_{k}$ are the complex amplitudes of the pulses and $\boldsymbol{\mu}$ is a fixed unit vector representing the basic pulse modulation. The parameters $\tau_{k}$ and $\omega_{k}$ are the circular time and frequency shifts required to align the $k$ th pulse with $\boldsymbol{\mu}$. The $\epsilon_{k}$ are independent circular complex Gaussian distributed random vectors satisfying $E(\boldsymbol{\epsilon})=\mathbf{0}$ and $E\left(\boldsymbol{\epsilon} \boldsymbol{\epsilon}^{H}\right)=\sigma^{2} \boldsymbol{I} . E(\cdot)$ denotes the expectation operator, $(\cdot)^{H} \mathrm{de}-$ notes the conjugate transpose, and $\sigma^{2}$ is the noise variance assumed to be known. $I$ is an $N_{S} \times N_{S}$ identity matrix. Note that the Gaussian distribution is chosen only as the nominal noise distribution.

The time and frequency shift operator matrices $\mathcal{T}(\tau)$ and $\boldsymbol{\Omega}(\omega)$ are given by [4]

$$
\begin{aligned}
\boldsymbol{\Omega}(\omega)_{k n} & =\exp (-j n \omega) \delta_{k n}, \\
\mathcal{T}(\tau) & =\mathcal{F}^{-1} \boldsymbol{\Omega}(2 \pi \tau) \mathcal{F},
\end{aligned}
$$

where $\delta_{k n}$ is the Kronecker delta, $j$ is the imaginary unit, and $\mathcal{F}$ denotes the discrete Fourier transform matrix

$$
\mathcal{F}_{k n}=\exp \left(-j 2 \pi k n / N_{S}\right) / \sqrt{N_{S}} .
$$

Before the pulse modulation is estimated, the pulses are preprocessed in order to align the pulses in time and frequency. In this work, the time alignment of the pulses after the preprocessing is assumed to be exact. However, as an extension to our previous work [5] the pulses are not assumed to be perfectly aligned in frequency. That is, the signal model after the preprocessing is assumed to be given by

$$
\boldsymbol{z}_{k}=A_{k} \boldsymbol{\Omega}\left(\nu_{k}\right) \boldsymbol{\mu}+\boldsymbol{\epsilon}_{k}^{\prime}, \quad k=1, \ldots, N_{P},
$$


where $\nu_{k}$ is the remaining frequency offset of the $k$ th pulse after the preprocessing. Since matrices $\mathcal{T}(\tau)$ and $\boldsymbol{\Omega}(\omega)$ are unitary, the noise distribution remains the same.

\section{PREPROCESSING}

In this work the preprocessing proposed in [5] is employed. First the carrier frequencies of the pulses are estimated using the Lank, Reed, Pollon frequency estimator given by [6]

$$
\hat{\omega}_{\boldsymbol{y}_{k}}=\angle \sum_{n=2}^{N_{S}} \boldsymbol{y}_{k}(n) \boldsymbol{y}_{k}^{*}(n-1)
$$

where $\angle$ denotes the phase angle, and $*$ denotes the complex conjugate. After carrier frequencies have been estimated, the pulses are transferred to the baseband

$$
\boldsymbol{z}_{k}(n)=\boldsymbol{\Omega}\left(\hat{\omega}_{\boldsymbol{y}_{k}}\right)^{H} \boldsymbol{y}_{k}, \quad k=1, \ldots, N_{P} .
$$

Carrier frequency offset $\nu_{k}=\omega_{k}-\hat{\omega}_{y_{k}}$ remains in the baseband pulses. Note that due to the symmetry of the matrices $\mathcal{T}(\tau)$ and $\boldsymbol{\Omega}(\omega)$ their order in (1) is interchangeable.

For the time alignment, the pulses $\boldsymbol{z}_{k}, k=1, \ldots, N_{P}$, are first rank ordered into a descending order based on signal-to-noise ratio (SNR), i.e. the pulse $\boldsymbol{z}_{1}$ has the largest SNR while the pulse $\boldsymbol{z}_{N_{P}}$ has the smallest SNR. Then the pulses are aligned in time according to the following procedure [5]:

For $k=2, \ldots, N_{P}$,

1. Calculate $c_{j}(\tau)=\left|\boldsymbol{z}_{j}^{H}(0) \boldsymbol{z}_{k}(\tau)\right|, \tau=0, \ldots, N_{S}-1$, for all $j=1, \ldots, k-1$. Here $z_{k}(\tau)$ denotes $\tau$ samples circularly shifted version of $\boldsymbol{z}_{k}$.

2. Time shift of the $k$ th pulse is given by $\hat{\tau}_{k}=\arg \max _{\tau} \sum_{j=1}^{k-1} c_{j}(\tau)$.

3. Shift the $k$ th pulse circularly $\hat{\tau}_{k}$ samples, i.e. $\boldsymbol{z}_{k}=$ $\boldsymbol{z}_{k}\left(\hat{\tau}_{k}\right)$.

At high SNR regime the computational complexity of the time alignment can be reduced by employing adaptive thresholding of the leading edge of the pulse [7] to obtain initial estimates for the time parameters $\tau_{k}$. Starting from the initial estimates the crosscorrelations in Step 1 of the above algorithm can be calculated only for small time shifts (i.e. a few samples left or right).

\section{M-ESTIMATION ALGORITHM}

The goal in the algorithm proposed in this section is to improve the frequency alignment of the pulses after preprocessing while maintaining robustness against preprocessing errors. Misaligned pulses cause large errors that are not well represented by the Gaussian noise model. M-estimators reduce the effect of large errors by minimizing a function of residual errors that bounds the influence of the large errors. In the following, an M-estimator that uses pulse weights (i.e. common weight parameter for all the samples in a pulse) for reducing the influence of misaligned pulses is derived.

The task is to find M-estimates for $\boldsymbol{\mu}, A_{k}$ 's, and $\nu_{k}$ 's. In the following this is realized by using a combination of an iterative reweighted least-squares (IRLS) procedure and SCG algorithm. The idea is to use SCG to update the $\nu_{k}$ 's, and at each iteration of the SCG to use the IRLS procedure to calculate the estimates for $\boldsymbol{\mu}$ and $A_{k}$ 's while keeping the $\nu_{k}$ 's fixed. The IRLS procedure to calculate the estimates for $\boldsymbol{\mu}$ and $A_{k}$ 's is given as follows ( $\nu_{k}$ 's are kept fixed)
1. Initialize weights $w_{k}=1, k=1, \ldots, N_{P}$, and set $i=0$.

2. Minimize the following weighted least-squares criterion

$$
\left.f=\sum_{k=1}^{N_{P}} w_{k} \| \boldsymbol{z}_{k}-A_{k} \boldsymbol{\Omega}\left(\nu_{k}\right) \boldsymbol{\mu}\right) \|^{2},
$$

in order to get $\hat{\boldsymbol{\mu}}^{(i+1)}$ and $\hat{A}_{k}^{(i+1)}, k=1, \ldots, N_{P}$.

3. Recalculate the weights

$$
w_{k}=w\left(\frac{\left\|\boldsymbol{z}_{k}-\hat{A}_{k}^{(i+1)} \boldsymbol{\Omega}\left(\nu_{k}\right) \hat{\boldsymbol{\mu}}^{(i+1)}\right\|}{\sigma \sqrt{N_{S}}}\right) .
$$

4. If the change in the error is small, stop, i.e., if

$$
L^{(i)} / L^{(i+1)}<1+\beta,
$$

where $L^{(i)}=\sum_{k=1}^{N_{P}}\left\|\boldsymbol{z}_{k}-\hat{A}_{k}^{(i)} \boldsymbol{\Omega}\left(\nu_{k}\right) \hat{\boldsymbol{\mu}}^{(i)}\right\|^{2}$ and $\beta$ is a small number, e.g. 0.001. Otherwise $i=i+1$ and go back to Step 2.

The $w(r)$ is called the weight function. The Huber weight function used in this work is given by [8]

$$
w(r)= \begin{cases}1, & \text { for }|r|<k \\ k /|r|, & \text { for }|r| \geq k,\end{cases}
$$

where $k$ is tuning constant. Value $k=1.345$ was experimentally selected for the simulations. The residual errors are normalized wrt scale $\sigma$, i.e. the standard deviation of the noise which is assumed to be known. If $\sigma$ is not known a simple and robust estimate of the scale is the MAD estimator [8].

In Step 2 of the above IRLS procedure, the task is to minimize the following weighted least-squares criterion

$f=\sum_{k=1}^{N_{P}} w_{k}\left(\left\|\boldsymbol{z}_{k}\right\|^{2}-\left|\boldsymbol{\mu}^{H} \boldsymbol{\Omega}\left(\nu_{k}\right)^{H} \boldsymbol{z}_{k}\right|^{2}+\left|A_{k}-\boldsymbol{\mu}^{H} \boldsymbol{\Omega}\left(\nu_{k}\right)^{H} \boldsymbol{z}_{k}\right|^{2}\right)$

From (10), it can be easily seen that $f$ is minimized wrt. $A_{k}$ by choosing

$$
\hat{A}_{k}=\boldsymbol{\mu}^{H} \boldsymbol{\Omega}\left(\nu_{k}\right)^{H} \boldsymbol{z}_{k}, \quad k=1, \ldots, N_{P} .
$$

A reduced cost function is obtained by substituting $A_{k}$ in (10) by $\hat{A}_{k}$

$$
f^{\prime}=\sum_{k=1}^{N_{P}} w_{k}\left(\left\|\boldsymbol{z}_{k}\right\|^{2}-\left|\boldsymbol{\mu}^{H} \boldsymbol{\Omega}\left(\nu_{k}\right)^{H} \boldsymbol{z}_{k}\right|^{2}\right) .
$$

Minimizing (12) wrt. $\boldsymbol{\mu}$ is equivalent to maximizing

$$
\begin{aligned}
\sum_{k=1}^{N_{P}} w_{k}\left|\boldsymbol{\mu}^{H} \boldsymbol{\Omega}\left(\nu_{k}\right)^{H} \boldsymbol{z}_{k}\right|^{2} & =\boldsymbol{\mu}^{H}\left(\sum_{k=1}^{N_{P}} w_{k} \boldsymbol{\Omega}\left(\nu_{k}\right)^{H} \boldsymbol{z}_{k} \boldsymbol{z}_{k}^{H} \boldsymbol{\Omega}\left(\nu_{k}\right)\right) \boldsymbol{\mu} \\
& =\boldsymbol{\mu}^{H} \boldsymbol{Z}_{\Omega} \boldsymbol{\mu} .
\end{aligned}
$$

The above expression is maximized if $\boldsymbol{\mu}$ is equal to the eigenvector of $\boldsymbol{Z}_{\Omega}$ corresponding to the largest eigenvalue [9, p. 176]. The eigenvector can be solved iteratively by employing the power method, i.e.

$$
\begin{aligned}
& \hat{\boldsymbol{\mu}}_{l+1}=\boldsymbol{Z}_{\Omega} \hat{\boldsymbol{\mu}}_{l}=\sum_{k=1}^{N_{P}} w_{k}\left(\boldsymbol{z}_{k}^{H} \boldsymbol{\Omega}\left(\nu_{k}\right) \hat{\boldsymbol{\mu}}_{l}\right) \boldsymbol{\Omega}\left(\nu_{k}\right)^{H} \boldsymbol{z}_{k}, \\
& \hat{\boldsymbol{\mu}}_{l+1}=\hat{\boldsymbol{\mu}}_{l+1} /\left\|\hat{\boldsymbol{\mu}}_{l+1}\right\| .
\end{aligned}
$$


What remains to be done, is to find the estimates for the $\nu_{k}$ 's. This is done by minimizing (10) with respect to $\nu_{k}$ 's. In order to do this, a gradient-based algorithm is employed. The gradient of (10) is

$\nabla_{\nu_{k}} f=\frac{\partial f}{\partial \nu_{k}}=-w_{k} A_{k}^{*} \boldsymbol{\mu}^{H} \frac{\partial \boldsymbol{\Omega}\left(\nu_{k}\right)^{H}}{\partial \nu_{k}} \boldsymbol{z}_{k}-w_{k} A_{k} \boldsymbol{z}_{k}^{H} \frac{\partial \boldsymbol{\Omega}\left(\nu_{k}\right)}{\partial \nu_{k}} \boldsymbol{\mu}$,

where the partial derivatives $\frac{\partial \boldsymbol{\Omega}\left(\nu_{k}\right)}{\partial \nu_{k}}$ are

$$
\frac{\partial \boldsymbol{\Omega}\left(\nu_{k}\right)_{k n}}{\partial \nu_{k}}=-j n \exp \left(-j n \nu_{k}\right) \delta_{k n}=-j n \boldsymbol{\Omega}\left(\nu_{k}\right)_{k n} .
$$

Now by denoting $\boldsymbol{\Omega}_{n}(\nu)_{k n}=n \boldsymbol{\Omega}(\nu)_{k n}$, it follows

$$
\begin{aligned}
\nabla_{\nu_{k}} f & =-j w_{k} A_{k}^{*} \boldsymbol{\mu}^{H} \boldsymbol{\Omega}_{n}\left(\nu_{k}\right)^{H} \boldsymbol{z}_{k}+j w_{k} A_{k} \boldsymbol{z}_{k}^{H} \boldsymbol{\Omega}_{n}\left(\nu_{k}\right) \boldsymbol{\mu}, \\
& =-2 \operatorname{Im}\left\{w_{k} A_{k} \boldsymbol{z}_{k}^{H} \boldsymbol{\Omega}_{n}\left(\nu_{k}\right) \boldsymbol{\mu}\right\}, \quad k=1, \ldots, N_{P},
\end{aligned}
$$

where $\operatorname{Im}\{\cdot\}$ denotes the imaginary part.

Now the Hessian is obtained as

$$
\nabla_{\nu_{k} \nu_{k}}^{2} f=\frac{\partial^{2} f}{\partial \nu_{k}^{2}}=2 \operatorname{Re}\left\{w_{k} A_{k} \boldsymbol{z}_{k}^{H} \boldsymbol{\Omega}_{n n}\left(\nu_{k}\right) \boldsymbol{\mu}\right\}, \quad k=1, \ldots, N_{P},
$$$$
\nabla_{\nu_{k} \nu_{i}}^{2} f=\frac{\partial^{2} f}{\partial \nu_{k} \partial \nu_{i}}=0, \quad \forall k \neq i,
$$

where $\operatorname{Re}\{\cdot\}$ denotes the real part, and $\boldsymbol{\Omega}_{n n}(\nu)_{k n}=n^{2} \boldsymbol{\Omega}(\nu)_{k n}$.

In this work the simple, yet effective, scaled conjugate gradient (SCG) algorithm [10] is employed for finding the optimum frequency vector $\boldsymbol{\nu}$ containing the unknown parameters $\nu_{k}, k=1, \ldots$, $N_{P}$. The SCG algorithm is briefly explained in the following.

The conjugate gradient methods minimize a differentiable function $f: \mathbb{R}^{N_{P}} \rightarrow \mathbb{R}$ iteratively as follows

$$
\boldsymbol{\nu}_{j+1}=\boldsymbol{\nu}_{j}+\lambda_{j} \boldsymbol{d}_{j},
$$

where $\boldsymbol{d}_{j}$ is the search direction and $\lambda_{j}$ is the step size which minimizes $f\left(\boldsymbol{\nu}_{j}+\lambda \boldsymbol{d}_{j}\right)$. For the first iteration, the negative gradient can be selected as the search direction, i.e. $\boldsymbol{d}_{1}=-\nabla f\left(\boldsymbol{\nu}_{1}\right)$. For the subsequent iterations, the search directions are

$$
\boldsymbol{d}_{j+1}=-\nabla f\left(\boldsymbol{\nu}_{j+1}\right)+\alpha_{j} \boldsymbol{d}_{j}
$$

where $\alpha_{j}$ is a suitable deflection parameter that characterizes a particular conjugate gradient method. The Polak-Ribiere conjugate gradient used in this work is given by [11, p. 329]

$$
\alpha_{j}=\frac{\nabla f\left(\boldsymbol{\nu}_{j+1}\right)^{T}\left(\nabla f\left(\boldsymbol{\nu}_{j+1}\right)-\nabla f\left(\boldsymbol{\nu}_{j}\right)\right)}{\left\|\nabla f\left(\boldsymbol{\nu}_{j}\right)\right\|^{2}} .
$$

The SCG algorithm avoids the time consuming line search procedure of the conventional conjugate gradient algorithms to find $\lambda_{j}$ in (21). In the SCG the step size $\lambda_{j}$ is obtained by minimizing a quadratic approximation to the function $f\left(\boldsymbol{\nu}_{j}+\lambda_{j} \boldsymbol{d}_{j}\right)$ with respect to $\lambda_{j}$. The step size is given by [10]

$$
\lambda_{j}=-\frac{\boldsymbol{d}_{j}^{T} \nabla f\left(\boldsymbol{\nu}_{j}\right)}{\boldsymbol{d}_{j}^{T} \boldsymbol{H} \boldsymbol{d}_{j}+\gamma_{j}\left\|\boldsymbol{d}_{j}\right\|^{2}}
$$

where $\boldsymbol{H}$ is the Hessian matrix and $\gamma_{j} \geq 0$ is sufficiently large diagonal loading factor that ensures the positive definiteness of the Hessian. That is, the Hessian matrix becomes $\boldsymbol{H}+\gamma_{j} \boldsymbol{I}$. For a detailed description of the selection of the value for $\gamma_{j}$ as well as description of the whole SCG algorithm, see [10].

A summary of the method for estimating a common modulation from a group of pulses using SCG is given below ( $\hat{\boldsymbol{A}}$ is a vector containing the amplitude estimates):
1. Initialize $\hat{\boldsymbol{\nu}}_{1}=\mathbf{0}$ and $\hat{\boldsymbol{\mu}}_{1}^{(1)}=\boldsymbol{z}_{\max } /\left\|\boldsymbol{z}_{\max }\right\|$ where $\boldsymbol{z}_{\max }$ is the pulse having the largest SNR.

2. Estimate $\hat{\boldsymbol{\mu}}_{1}$ and $\hat{\boldsymbol{A}}_{1}$ using the IRLS procedure.

3. Initialize $\boldsymbol{d}_{1}=-\nabla f\left(\hat{\boldsymbol{A}}_{1}, \hat{\boldsymbol{\nu}}_{1}, \hat{\boldsymbol{\mu}}_{1}\right)$ using (18).

4. Update $\hat{\boldsymbol{\nu}}_{j+1}=\hat{\boldsymbol{\nu}}_{j}+\lambda_{j} \boldsymbol{d}_{j}$ where $\lambda_{j}$ is given by (24).

5. Estimate $\hat{\boldsymbol{\mu}}_{j+1}$ and $\hat{\boldsymbol{A}}_{j+1}$ using the IRLS procedure. Initialization $\hat{\boldsymbol{\mu}}_{j+1}^{(1)}=\boldsymbol{\Omega}\left(\hat{\nu}_{\max , j+1}\right)^{H} \boldsymbol{z}_{\max } /\left\|\boldsymbol{\Omega}\left(\hat{\nu}_{\max , j+1}\right)^{H} \boldsymbol{z}_{\max }\right\|$ where $\hat{\nu}_{\max , j+1}$ is the current estimate of the frequency offset of pulse $\boldsymbol{z}_{\max }$.

6. Update $\boldsymbol{d}_{j+1}=-\nabla f\left(\hat{\boldsymbol{A}}_{j+1}, \hat{\boldsymbol{\nu}}_{j+1}, \hat{\boldsymbol{\mu}}_{j+1}\right)+\alpha_{j} \boldsymbol{d}_{j}$ where $\alpha_{j}$ is given by (23).

7. If converged stop (i.e. if the change in the error or in the search direction is smaller than a predefined threshold). Otherwise go to Step 4.

During the running of the SCG algorithm, the conjugacy of the search directions tends to deteriorate. Therefore, it is recommended to restart the algorithm after every $N_{P}$ steps. That is, the search vector is reset to the negative gradient direction after every $N_{P}$ steps.

Note that each time the IRLS procedure is used, the weights are initialized to one and $\boldsymbol{\mu}$ is initialized to the pulse having the largest SNR (with the current frequency alignment estimate). This strategy was chosen in order to avoid getting stuck in local minima.

\section{POSTPROCESSING}

The Lank, Reed, Pollon carrier frequency estimator in combination with the SCG algorithm can result in a small residual carrier frequency offset in the final pulse modulation estimate. In addition, the time location of the pulse within the pulse vector can vary. In order to improve the identification performance the following postprocessing steps are proposed.

The alignment for comparison of two pulse modulation estimates $\hat{\boldsymbol{\mu}}_{1}$ and $\hat{\boldsymbol{\mu}}_{2}$ proceeds as follows. First the estimates $\hat{\boldsymbol{\mu}}_{1}$ and $\hat{\boldsymbol{\mu}}_{2}$ are aligned in time domain based on cross-correlation (i.e. time shift giving the largest cross-correlation is chosen). Then, in order to align the estimates $\hat{\boldsymbol{\mu}}_{1}$ and $\hat{\boldsymbol{\mu}}_{2}$ in frequency domain, the following cost function is minimized wrt. $\omega$

$$
1-\left|\hat{\boldsymbol{\mu}}_{1}^{H} \boldsymbol{\Omega}(\omega)^{H} \hat{\boldsymbol{\mu}}_{2}\right|^{2},
$$

using quadratic fit line search algorithm [11, p. 280].

In the simulation experiments $\hat{\boldsymbol{\mu}}_{1}$ is the true modulation profile and $\hat{\boldsymbol{\mu}}_{2}$ is the obtained estimate.

\section{SIMULATION RESULTS}

The performance of the proposed M-estimation technique is investigated in this section using simulation experiments. The performance is compared to the ML [4] and M-estimation [5] methods. All the methods use the pre- and postprocessing procedures presented in this paper. Two test pulses are used. The first pulse is a linear frequency modulated (LFM) pulse given by

$\boldsymbol{\mu}_{0}(n)=\exp \left(j 2 \pi\left(-\frac{1}{24} n+\frac{125}{432} \times 0.001 n^{2}\right)\right), n=0, \ldots, 143$

The second pulse has a sinusoidal frequency modulation (FM) (i.e. nonlinear frequency modulation) given by

$\boldsymbol{\mu}_{0}(n)=\exp \left(j 2 \pi \times 0.02 \sum_{k=0}^{n} \sin (2 \pi k / 140)\right), n=0, \ldots, 120$. 


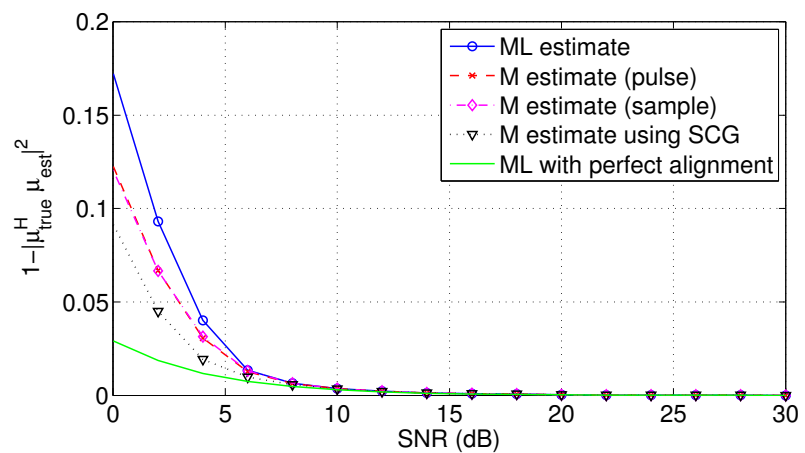

(a) LFM

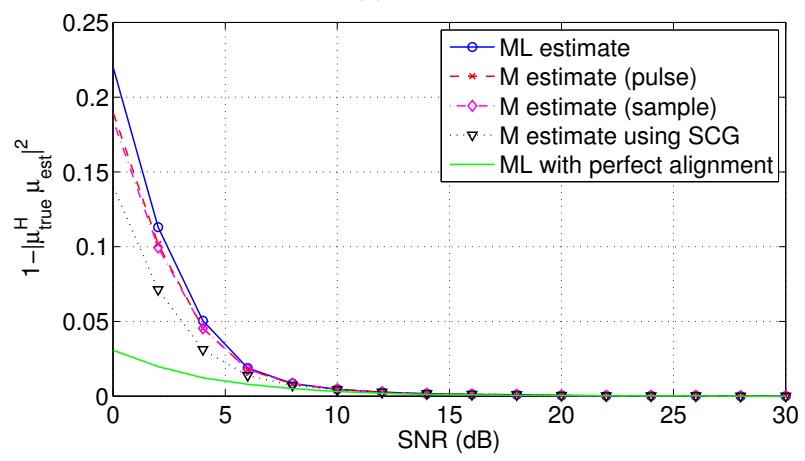

(b) Sinusoidal FM

Fig. 1. $\left(1-\left|\boldsymbol{\mu}_{\text {true }}^{H} \hat{\boldsymbol{\mu}}\right|^{2}\right)$ vs. SNR for the ML and M-estimators for 30 (a) LFM and (b) sinusoidal FM pulses. The M-estimator using SCG has better performance at low SNR regime due to improved frequency alignment of the pulses.

The norms of the pulses are normalized to one. Both pulses have rise and fall times of $1 / 12$ and $1 / 4$ of the pulse length, respectively. Raised cosine model is used for pulse shaping.

Figs. 1 and 2 depict the estimation performance as a function of the SNR and number of intercepted pulses, respectively. The curves are averages over 500 simulation experiments.

\section{CONCLUSION}

In this paper a robust M-estimation technique for estimating a common modulation from a group of intercepted pulses was proposed. The proposed technique uses SCG algorithm for improving the frequency alignment of the pulses. Simulation experiments showed that the proposed technique provides better performance at low SNR regime than the previously proposed ML and M-estimation techniques. This is due to the better frequency alignment of the pulses obtained by the use of SCG algorithm.

\section{REFERENCES}

[1] J. Liu, J. P. Y. Lee, L. Li, Z.-Q. Luo, and K. M. Wong, "Online clustering algorithms for radar emitter classification," IEEE Trans. Pattern Anal. Machine Intell., vol. 27, no. 8, pp. 11851196, Aug. 2005.

[2] A. Kawalec and R. Owczarek, "Specific emitter identification using intrapulse data," in European Radar Conference 2004, Amsterdam, Oct. 2004, pp. 249-252.

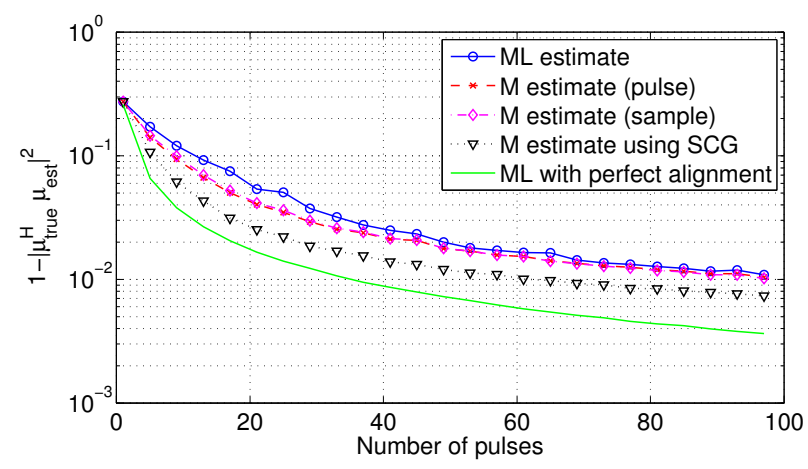

(a) LFM

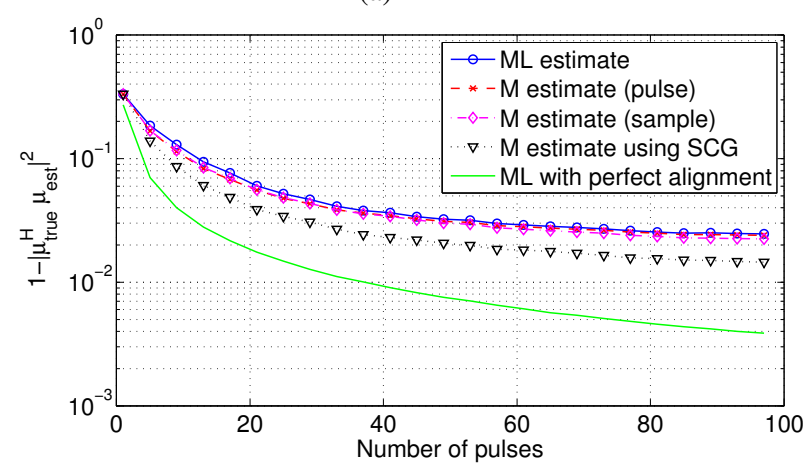

(b) Sinusoidal FM

Fig. 2. $\left(1-\left|\boldsymbol{\mu}_{\text {true }}^{H} \hat{\boldsymbol{\mu}}\right|^{2}\right)$ vs. number of pulses for the ML and Mestimators for (a) LFM and (b) sinusoidal FM pulses with SNRs between 3 and $5 \mathrm{~dB}$. The M-estimator using SCG has better performance due to better frequency alignment of the pulses. However, for the sinusoidal FM the gap to ML with perfect alignment is still quite large.

[3] B. W. Gillespie and L. E. Atlas, "Optimizing time-frequency kernels for classification," IEEE Trans. Signal Processing, vol. 49, no. 3, pp. 485-496, Mar. 2001.

[4] S. D. Howard, "Estimation and correlation of radar pulse modulations for electronic support," in Proc. IEEE Aerospace Conference 2003, Mar. 8-15, 2003, vol. 5, pp. 2065-2072.

[5] J. Lundén and V. Koivunen, "Robust estimation of radar pulse modulation," in Proc. 6th IEEE Int. Symp. on Signal Processing and Inform. Technology, Vancouver, Aug. 27-30, 2006.

[6] G. W. Lank, I. S. Reed, and G. E. Pollon, "A semicoherent detection and doppler estimation statistic," IEEE Trans. Aerosp. Electron. Syst., vol. AES-9, pp. 151-165, Mar. 1973.

[7] D. J. Torrieri, "Statistical theory of passive location systems," IEEE Trans. Aerosp. Electron. Syst., vol. 20, pp. 183-198, Mar. 1984.

[8] P. J. Huber, Robust statistics, John Wiley \& Sons, New York, 1981.

[9] R. Horn and C. Johnson, Matrix analysis, Cambridge, 1985.

[10] M. F. Møller, "A scaled conjugate gradient algorithm for fast supervised learning," Neural Networks, vol. 6, no. 4, pp. 525533, 1993.

[11] M. S. Bazaraa, H. D. Sherali, and C. M. Shetty, Nonlinear programming: theory and algorithms, 2 nd ed., John Wiley \& Sons, 1993. 